\title{
BMJ Global Health Barriers and facilitators influencing facility-based childbirth in rural Haiti: a mixed method study with a convergent design
}

\author{
Maxi Raymondville, ${ }^{1,2}$ Carly A Rodriguez, ${ }^{1}$ Aaron Richterman,, ${ }^{3,4}$ Gregory Jerome, ${ }^{2}$ \\ Arlene Katz, ${ }^{1}$ Hannah Gilbert, ${ }^{1}$ Gregory Anderson, ${ }^{2}$ Jean Paul Joseph, ${ }^{2}$ \\ Molly F Franke, ${ }^{1}$ Louise C Ivers (D) ${ }^{1,4}$
}

To cite: Raymondville $\mathrm{M}$, Rodriguez CA, Richterman A, et al. Barriers and facilitators influencing facility-based childbirth in rural Haiti: a mixed method study with a convergent design. BMJ Global Health 2020;5:e002526. doi:10.1136/ bmjgh-2020-002526

Handling editor Seye Abimbola

MFF and LCl are Co-last authors.

Received 23 March 2020

Revised 3 July 2020

Accepted 5 July 2020

Check for updates

(C) Author(s) (or their employer(s)) 2020. Re-use permitted under CC BY-NC. No commercial re-use. See rights and permissions. Published by BMJ.

${ }^{1}$ Department of Global Health and Social Medicine, Harvard Medical School, Boston, MA, USA

2Zanmi Lasante, Partners In Health, Port-au-Prince, Haiti ${ }^{3}$ Division of Infectious Diseases, Brigham and Women's Hospital, Boston, MA, USA

${ }^{4}$ Center for Global Health, Massachusetts General Hospital Boston, Massachusetts, USA

Correspondence to Dr Maxi Raymondville; maxhaiti@aol.com

\section{ABSTRACT}

Introduction Haiti has the highest maternal mortality rate in the Western Hemisphere. Facility-based childbirth is promoted as the standard of care for reducing maternal and neonatal mortality. We conducted a convergent, mixed methods study to assess barriers and facilitators to facilitybased childbirth at Hôpital Universitaire de Mirebalais (HUM) in Mirebalais, Haiti.

Methods We conducted secondary analyses of a prospective cohort of pregnant women seeking antenatal care at HUM and quantitatively assessed predictors of not having a facility-based childbirth at HUM. We prospectively enrolled 30 pregnant women and interviewed them about their experiences delivering at home or at HUM.

Results of 1105 pregnant women seeking antenatal care at the hospital between May and December 2017, $773(70 \%)$ returned to the hospital for facility-based childbirth. In multivariable analyses, living farther from the hospital (adjusted OR (AOR) $=0.73 ; 95 \% \mathrm{Cl} 0.56$ to 0.96), poverty ( $\mathrm{AOR}=0.93 ; 95 \% \mathrm{Cl} 0.88$ to 0.99 ) and household hunger ( $\mathrm{AOR}=0.45 ; 95 \% \mathrm{Cl} 0.26$ to 0.79 ) were associated with not having a facility-based childbirth. Primigravid women were more likely to have a facility-based childbirth ( $A 0 \mathrm{R}=1.34,95 \% \mathrm{Cl} 1.02$ to 1.76). Qualitative data provided insight into the value women place on traditional birth attendants ('matrons') during home-based childbirths. While women perceived facility-based childbirths as better equipped to handle birth complications, barriers such as distance, costs of transportation and supplies, discomfort of facility birthing practices and mistreatment by medical staff resulted in negative perceptions of facility-based childbirths.

Conclusion Pregnant women in rural Haiti must overcome substantial structural barriers and forfeit valued support from traditional birth attendants when they pursue facility-based childbirths. If traditional birth attendants could be involved in care alongside midwives at facilities, women may be more inclined to deliver there. While complex structural barriers remain, the inclusion of matrons at facilities may increase uptake of facility-based childbirths, and ultimately improve maternal and neonatal outcomes.

\section{Key questions}

What is already known?

- Haiti has the highest maternal mortality rate in the Western Hemisphere.

- Despite facility-based childbirth being the standard of care for reducing maternal and neonatal mortality, an estimated $63 \%$ of Haitian women give birth at home.

What are the new findings?

- Of 1105 pregnant women seeking antenatal care at Hôpital Universitaire de Mirebalais (HUM) in Mirebalais, Haiti, 773 (70\%) returned to the hospital for facility-based childbirth.

- Not returning to the hospital for birth was associated with living further, poverty, household hunger and having had prior births.

- Women described how they thought facility-based births were safer, but barriers such as distance, cost of transportation and supplies, discomfort with facility birthing practices and mistreatment by medical staff negatively affected their decision to deliver at the facility.

- Women described deep appreciation for the physica and spiritual support provided during delivery by traditional birth attendants ('matrons').

What do the new findings imply?

- Pregnant women in rural Haiti must overcome substantial structural barriers related to poverty and forfeit valued support from traditional birth attendants when they pursue facility-based childbirths.

- The presence of the matron in the delivery room alongside midwives may facilitate increased comfort and support for women, thereby encouraging pregnant women to pursue facility-based deliveries.

\section{INTRODUCTION}

Haiti has the highest maternal mortality rate in the Western Hemisphere, with an estimated 359 maternal deaths per 100000 live births. ${ }^{1}$ While progress has been made, Haiti did not 
achieve the United Nations Millennium Development Goal (MDG) of decreasing maternal mortality by $75 \%$ by $2015 .^{2}$ One potential reason may be because an estimated $63 \%$ of Haitian women give birth at home. ${ }^{3}$ Facility-based childbirth is the standard of care for reducing maternal and neonatal mortality because skilled birth attendants can recognise emergency obstetrical complications and provide timely management and treatment, including caesarean section. ${ }^{4}{ }^{5}$ In 1994, Thaddeus and Maine published a framework introducing the 'three delays' preventing pregnant women from delivering in a facility. The 'three delays' include factors that (1) delay the decision to seek care, (2) delay arriving at a health facility and (3) delay provision of adequate care. ${ }^{6}$ While causes of these delays have been investigated, ${ }^{7-12}$ few studies have assessed this framework in the context of rural Haiti. ${ }^{13-15}$

Tremendous gains in the quality of care for women's health are needed to meet the Sustainable Development Goals (SDGs) target of 70 maternal deaths per 100000 live births by $2030 .^{2}$ The Haitian Ministry of Health and international agencies suggest improving infrastructure quality, hiring more staff and securing supplies and drugs may increase uptake of facility-based childbirths. However, barriers to facility-based childbirth must be better understood to inform efforts to reach the SDG target. With the goal of increasing facility-based childbirths and improving care for pregnant women and their infants, we studied barriers and facilitators to facilitybased childbirth at Hôpital Universitaire de Mirebalais (HUM), a district hospital in rural Haiti. Specifically, we sought to understand the role of traditional birth attendants, distance, access to transport and perceptions of quality of care received at the district hospital.

\section{METHODS}

\section{Study design and setting}

We conducted a mixed method study using a convergent design. ${ }^{16}$ The purpose of a convergent mixed methods design is to collect both quantitative and qualitative data and directly compare, or 'converge,' the findings to determine whether the data are similar or dissimilar. To assess barriers and facilitators to facility-based childbirth at HUM, we conducted secondary analyses of a prospective cohort study of 1713 pregnant women aimed at investigating the impact of the Zika virus on pregnancy and neonatal outcomes. Women were eligible for inclusion if they attended at least one antenatal care (ANC) visit at HUM between May to December 2017 , were $\geq 16$ years, pregnant with laboratory confirmation and resided within the local municipality of Mirebalais where HUM is located. HUM is a 300-bed public teaching hospital managed by Zanmi Lasante (Partners In Health-Haiti). HUM's primary catchment area encompasses $>180000$ people seeking routine care and 3.1 million people seeking tertiary-level care. HUM's obstetric care capacity includes six operating theatres, ultrasound services, five obstetricians and gynaecologists and six nurse-midwives. The hospital performs approximately 5000 deliveries a year free of charge. In order to gain a deeper understanding of the lived experiences, perceptions, beliefs and desires around decision making for a facility-based childbirth, we conducted semi-structured interviews with 30 women who recently delivered and had an antenatal visit at HUM during the prospective cohort enrolment period. Participants received a transportation stipend of 160 Haitian Gourdes (approximately US $\$ 1.90$ ) at enrolment and again if they returned for delivery.

\section{Data collection}

\section{Quantitative data}

We collected data on participants' age, locality of residence, history of alcohol use and obstetrical history (number of prior pregnancies, live births, stillbirths, miscarriages and abortions) using a structured survey instrument and medical records. We calculated the distance from the centre of the participant's locality of residence to HUM. Using a previously validated poverty scorecard based on 11 indicators specific to Haiti, we estimated the likelihood a participant's household income was below the national poverty line of 83.39 Haitian Gourdes per day (approximately US $\$ 1$ ). ${ }^{17}$ The poverty scorecard included indicators such as the number of household members and their working status, building material of the home and source of water and energy for cooking. Higher poverty scores indicate a lower likelihood of living below the poverty line. We assessed food insecurity as an alternative indicator for poverty. We used the Household Hunger Scale, a three-item householdlevel measure of food access that has been validated across settings. ${ }^{18}$ These questions pertained to how often (eg, never, rarely, sometimes, often) in the last 4 weeks that there was no food to eat of any kind in the household, the respondent went to sleep hungry or the respondent went a whole day and night without eating.

\section{Qualitative data}

We collected qualitative data from July 2018 through January 2019. Using hospital registries, we identified women who had at least one ANC visit and purposively sampled 15 women who did not return to deliver at HUM and 15 women who gave birth at HUM. A Haitian obstetrician and gynaecologist from HUM (MR) conducted in person, individual interviews about participants' experiences during pregnancy and delivery. Interviews were conducted in Haitian Creole, lasted between 60 and $90 \mathrm{~min}$, and were conducted in a private space in women's homes or in the facility. Interviews followed a semi-structured interview guide including the following topics: (1) experiences of pregnancy and ANC, (2) plans and preferences for delivery, (3) benefits and drawbacks to home and facility deliveries and (4) experiences navigating the healthcare system. 


\section{Data analysis}

\section{Quantitative data}

We calculated descriptive statistics and used univariable and multivariable logistic regression to identify predictors of not having a facility-based childbirth at HUM. We hypothesised that older maternal age, farther geographic proximity to HUM and poverty would decrease the odds of facility-based delivery. Likewise, we hypothesised that primigravity and prior negative child birth outcomes (number of prior miscarriages, stillbirths) would increase the odds of facility-based delivery. Of these predictors identified a priori, we included those associated with facility-based childbirth at a cut-off of $\mathrm{p}<0.20$ in multivariable logistic regression analyses.

We unintentionally omitted one indicator on the poverty scorecard during the first several months of the study. As a result, the calculated poverty score is missing for $31 \%$ of the participants. We used the missing-indicator method to account for missingness of the poverty score. ${ }^{19}$

\section{Qualitative data}

For qualitative analysis, we used an inductive, contentfocused approach with category construction, comparison and interpretation. ${ }^{20} \mathrm{MR}$ transcribed the interviews, and reviewed their contents. A subset of transcripts were open coded by MR and AK in order to identify concepts that were relevant to the research question. These concepts were formalised into a codebook by MR and HG, which was then piloted and revised. MR used the codebook to manually code the entire dataset. Coded data were inductively analysed in order to develop a set of initial conceptual categories that were labelled, described and illustrated with excerpts from the data. Initial categories were revised through an iterative process, resulting in a set of five final descriptive categories.

\section{Ethics statement}

Women enrolled in the quantitative study provided written informed consent. For qualitative interviews, an informed consent script was read verbatim to all potential participants, who provided oral consent. To maximise privacy, interview participants were encouraged to choose the date, time and place they felt most comfortable being interviewed. Participants were advised they could stop the interview at any time, and the interviewer was prepared to end the interview if participants appeared unwilling or uncomfortable. All procedures respected the confidentiality of the interviewees.

\section{Patient and public involvement}

This research was done without patient involvement. Patients were not invited to comment on the study design, consulted to develop patient relevant outcomes, interpret the results or invited to contribute to writing or editing.

\section{RESULTS}

\section{Quantitative results}

\section{Participant characteristics}

One thousand seven hundred thirteen pregnant women attended at least one ANC visit at HUM between May and December 2017. Because the analysis was restricted to participants from Mirebalais, 1105 (67\%) participants were included. Women were a mean of 28 years of age (SD: 6; range 16-50 years; table 1). Over a third of women were seeking care for their first pregnancy $(n=382,35 \%)$. Six per cent of women lived $>10 \mathrm{~km}$ from HUM. The mean likelihood of poverty was 36\% (SD 27) and 404 $(37 \%)$ women came from households with severe food insecurity. Seven hundred seventy-three women (70\%) returned to HUM for delivery; $332(30 \%)$ delivered at home or in another hospital. Of those who delivered at HUM, 132 (21\%) had a caesarean section.

\section{Predictors of not returning to HUM for facility-based childbirth}

In univariable analyses, women living in Gascogne (the communal section furthest from HUM and separated from HUM by a river) were less likely to deliver at HUM $(\mathrm{OR}=0.45 ; 95 \%$ CI 0.26 to $0.77 ; \mathrm{p}=0.003)$, relative to those who lived in Crete Brulee (the communal section closest to HUM) (table 2). Living $>10 \mathrm{~km}$ from the hospital reduced the odds of HUM birth $(\mathrm{OR}=0.53$; $95 \%$ CI 0.32 to $0.86 ; \mathrm{p}=0.01$ ); however, all of the women who lived $>10$ $\mathrm{km}$ from HUM lived in Gascogne. Women with a higher likelihood of poverty had lower odds of delivering at HUM (OR $=0.91$ per $10 \%$ increase in likelihood; $95 \%$ CI 0.86 to $0.96 ; \mathrm{p}=0.001)$. Severe hunger in the household was also associated with reduced odds of HUM delivery (OR $0.71,95 \%$ CI 0.54 to $0.91 ; \mathrm{p}=0.008$ ). Age, primigravity, prior stillbirth, prior miscarriage and prior abortion were not associated with HUM delivery.

Communal section, first pregnancy, prior abortion, likelihood of poverty and severe household food insecurity were included in the multivariable model (table 2). Primigravity was independently associated with an increased odds of returning to HUM for delivery (adjusted OR (AOR) 1.34, 95\% CI 1.02 to $1.76 ; \mathrm{p}=0.04$ ). Factors independently associated with decreased odds of HUM delivery included residing in Gascogne $(\mathrm{AOR}=0.45$; 95\% CI 0.26 to $0.79 ; \mathrm{p}=0.005)$, higher likelihood of poverty (AOR 0.93 per $10 \%$ increased likelihood; $95 \% \mathrm{CI}$ 0.88 to $0.99 ; \mathrm{p}=0.01$ ) and severe household food insecurity (AOR $0.73,95 \%$ CI 0.56 to $0.96, \mathrm{p}=0.02$ ).

\section{Qualitative results}

Five main themes emerged from qualitative interviews.

The matron (traditional birth attendant) as 'accompagnateur' Women appreciate the comfort and care provided by matrons during home-based childbirths

Participants' descriptions suggest that matrons assume a role of 'accompagnateur'"21 (ie, a trusted companion who supports one on her journey), providing both emotional and physical support to women during delivery at 
Table 1 Descriptive characteristics of Haitian women who resided in the Mirebalais commune $(n=1105$, unless specified otherwise)

\begin{tabular}{|c|c|c|c|c|c|c|}
\hline & \multicolumn{2}{|c|}{$\begin{array}{l}\text { Total population } \\
(n=1105)\end{array}$} & \multicolumn{2}{|c|}{$\begin{array}{l}\text { Delivered at HUM } \\
(n=773)\end{array}$} & \multicolumn{2}{|c|}{$\begin{array}{l}\text { Did not deliver a } \\
\text { HUM }(n=332)\end{array}$} \\
\hline & $\mathbf{N}$ & $\%$ & $\mathbf{n}$ & $\%$ & $\mathbf{n}$ & $\%$ \\
\hline \multicolumn{7}{|l|}{ Communal section } \\
\hline Crete Brulee & 317 & 29 & 235 & 30 & 82 & 25 \\
\hline Gascogne & 69 & 6 & 38 & 5 & 31 & 9 \\
\hline Grand Boucan & 389 & 35 & 275 & 36 & 114 & 34 \\
\hline Sarazin & 330 & 30 & 225 & 29 & 105 & 32 \\
\hline \multicolumn{7}{|l|}{ Age category (years) } \\
\hline$<20$ & 127 & 11 & 90 & 12 & 37 & 11 \\
\hline 20-34 & 785 & 71 & 543 & 70 & 242 & 73 \\
\hline$>34$ & 193 & 17 & 140 & 18 & 53 & 16 \\
\hline Distance between home and HUM (km), mean (SD) & 8.4 & 1.1 & 8.3 & 1.1 & 8.5 & 1.3 \\
\hline First pregnancy & 382 & 35 & 281 & 36 & 101 & 30 \\
\hline Prior abortion (one or more) & 48 & 4 & 39 & 5 & 9 & 3 \\
\hline Prior miscarriage (one or more) & 87 & 8 & 62 & 8 & 25 & 8 \\
\hline Prior stillbirths (one or more) & 107 & 10 & 72 & 9 & 35 & 11 \\
\hline \multicolumn{7}{|l|}{ Living children } \\
\hline 0 & 440 & 40 & 325 & 42 & 115 & 35 \\
\hline 1 & 557 & 50 & 376 & 49 & 181 & 55 \\
\hline 2 or more & 108 & 10 & 72 & 9 & 36 & 11 \\
\hline \multicolumn{7}{|l|}{ Prior livebirths } \\
\hline 0 & 431 & 39 & 317 & 41 & 114 & 34 \\
\hline 1 & 548 & 50 & 375 & 49 & 173 & 52 \\
\hline 2 or more & 126 & 11 & 81 & 10 & 45 & 14 \\
\hline Likelihood of poverty, mean (SD) $(n=766)$ & 36 & 27 & 33 & 26 & 41 & 27 \\
\hline \multicolumn{7}{|l|}{ Household hunger scale } \\
\hline No hunger in household & 377 & 34 & 274 & 35 & 103 & 31 \\
\hline Moderate hunger in household & 324 & 29 & 237 & 31 & 87 & 26 \\
\hline Severe hunger in household & 404 & 37 & 262 & 34 & 142 & 43 \\
\hline
\end{tabular}

HUM, Hôpital Universitaire de Mirebalais.

home. One participant described how matrons worked seamlessly with family members (eg, mother, motherin-law, sister or cousin) to literally hold up and support labouring women. Women described feeling comforted by that support, citing it as an advantage to giving birth at home. One participant described her perception of how women feel when giving birth at home:

Some of them are more comfortable at home because they are family members who touch their belly or doing massage at home and they have the matrons who are doing massage for them. (Home delivery)

Another participant spoke about the 'strength' she derives from the matron's emotional and physical support:

The matron touches you and asks you to push. In the hospital, they tell you to push too but it's when they see the baby arrive. The matron gives you strength. That's one of the reasons I love giving birth at home. (Home delivery)

Women explained that matrons provide holistic practices-outside the realm of standard obstetric care-that are important to the birthing experience. These practices provide women with a familiar and comforting environment for giving birth. In some cases, this involved the provision of teas to provide labouring women with extra 'strength'.

For the tea, she [matron] boiled orange leaves, after that she mix different types of leaves, then she put a leaf called 'Fobazin' in it and then she boils it and gives it to you to drink. It is so that you can have strength. Like if you lack strength, the tea will help you. (Hospital delivery)

Women also referenced prayers and rituals they associated with childbirth, which are only performed by 
Table 2 Univariable and multivariable analysis of predictors of in-hospital delivery among Haitian women with at least one antenatal visit ( $n=1105$, unless specified otherwise)

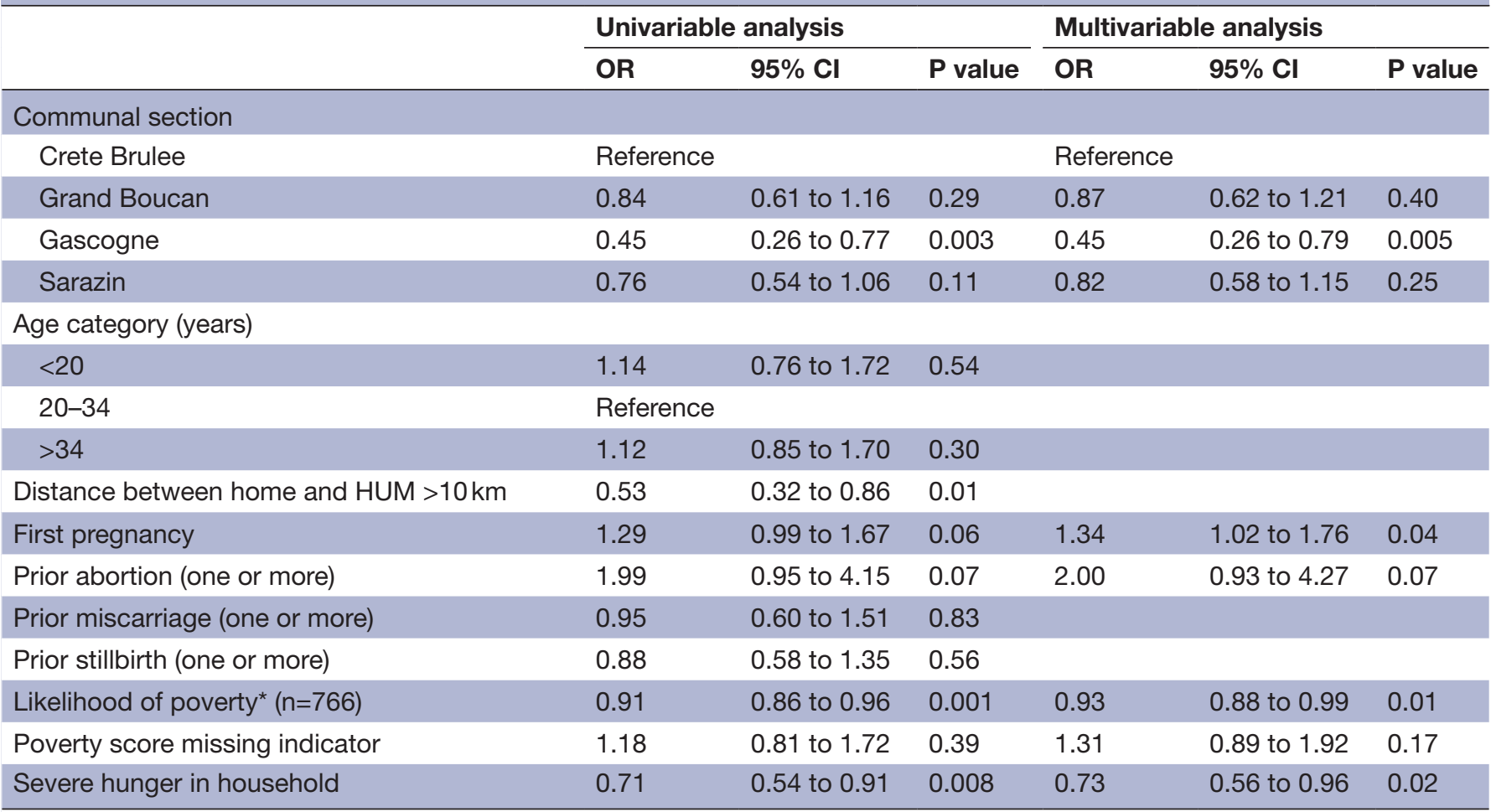

${ }^{*}$ Per $10 \%$ increase.

HUM, Hôpital Universitaire de Mirebalais.

matrons. One such ritual involves care of the placenta after birth:

The matron digs a hole and buries the umbilical cord. After that, they gave me a warm bath with papaya leaves and she gave me ginger tea to drink. [...] Sometimes the matron made a hole in front of the bed and put the placenta in there. Finally she burned it, after she tied the rest of the baby's umbilical cord, after she made prayers. (Home delivery)

Access to facility-based childbirths is shaped by distance and geographic access

The problem of infrastructure: distance and geographic access

Patients face limited public infrastructure in remote, rural villages. Haitian women living in rural areas must walk on secondary roads to reach paved roads leading to the hospital. Secondary roads extend outward from villages and are difficult to traverse due to hills, mud, rocks and overgrowth. One woman explained the number of hours she spent on the road before reaching the hospital:

[...] I spent 2 hours walking from my house to the paved road and from the paved road it takes me another 2 hours to reach the hospital on autobus. (Home delivery)

During the rainy season, rivers and ravines can flood and block river passage. There are no bridges and crossing flooded rivers is dangerous. Ambulances, when available, also cannot cross flooded rivers to retrieve labouring women. A woman explained how these conditions forced her to give birth at home:
I have 4 children who were born at home [...], but the 3 children were born in Pouille (Boucan Carre) with the help of matron Simene Casseus. Pouille is in principle close to Mirebalais, but you have to cross the river. When the level of the river is high, there is no way to cross and pregnant women are blocked on the other side. (Home delivery)

Women may go into labour in the middle of the night when it is particularly difficult to find transportation or someone to accompany her to the hospital. Nighttime travel can be risky in Haiti and it can be difficult to find people willing to provide transport. This causes women to defer hospital travel to the next day. Numerous women spoke about how their labour progressed quickly during the night:

I was doing preparations to move to another house when I had contractions. ... and I did not have a chance to go to the hospital. (Delivered on the road)

I spent all night with contractions and nobody wanted to give me a ride to go to the hospital, no motorcycle was available at this time. I did not have enough money to rent a car. I got the pains during the night and I have to call the neighborhood and I had to stay until the morning and try to get money to reach the hospital. (Hospital delivery)

Hidden costs associated with hospital delivery

Preparing for a newborn

Economic factors influence women's choices on where they give birth. Although there are no user fees at HUM, 
women may struggle to find money for costs associated with hospital delivery, such as providing baby linens (eg, layettes, towels, clothes and toiletries) and other items for comfort during their hospital stay. Women described how the arrival of the baby comes with expenses:

We could not do big, you know. I could not buy a suitcase, but we had prepared a small suitcase; we buy small things (linens) and we wash them, we iron them. If you will need a sheet to sleep on the floor, you will need to think about that. We cannot afford to buy a lot of things. We buy small things like those that the poor can buy because we don't have money. (Hospital delivery)

When you're poor, you handle your poverty. Not too much means. But you know, when you're poor and you're pregnant, you know all the things you are going to need but you cannot afford them. Even if the father has to go sacrifice himself, he gives me what I need. He gives me the money to go buy the things I need. (Hospital delivery)

\section{Hardship for food}

Women explained that in contrast to home deliveries, where food was available, families anticipating deliveries at HUM needed money to buy food for accompanying family members. While HUM provided some food to women, it would often be late, and was perceived by some participants as insufficient to support the physical work of labour:

We received food at the hospital, but the meal was not ready on time. (Hospital delivery)

\section{Costs for transportation}

Women explained how they walked from their homes across mountainous terrain to paved roads where they could access a car or motorcycle. Women explained motorcycles were not appropriate for transporting labouring women in pain or women discharged from the hospital after delivery. Cars were cited as particularly expensive and could cost US\$2 to US\$6. Notably, women reported drivers would inflate the cost in emergenciessometimes doubling or tripling the fare. One woman explained her journey:

I pay fifty gourdes [approximately 0.5 USD] to go and fifty gourdes to return. As long as it's the paved road, you pay fifty gourdes. But if it's from the village to the paved road, you can pay up to one hundred gourdes. (Hospital delivery)

\section{Facility-based practices create a negative environment} Women experienced discomfort as a result of hospital procedures Women frequently reported feeling uneasy or uncomfortable labouring on the delivery table, referred to as the 'ti bourik', or the small donkey. Women explained that their position on the table was uncomfortable. One woman explicitly contrasted the experience of delivering on the table with the position she was able to adopt when giving birth at home where she would be physically held by the matron or family members in comfort and privacy:
I do not like the delivery table, but I am obliged, I do not like the position that we must take the head down and feet in the air and when we are on this table, we must do whatever it takes to give birth. But at home it's not the same, you always find someone to support you. (Hospital delivery)

In the delivery room, since that's where I spent all my time because there wasn't any room, and I had to stay on the 'ti bourik' (small donkey=delivery table) where I delivered my baby, they kept me on a bed a little higher but it was still the delivery bed, I had to stay on one. (Hospital delivery)

\section{Lack of space and beds at HUM}

Following delivery, women are typically transferred to a bed to recover; however, women described that these beds were seldom available, which interfered with their ability to recuperate. Several women explained that shortly after delivering, they were informed there were no beds for them and they would need to recuperate with their newborns on the floor:

The women who came to deliver after me, they had to be on the floor... there were people who were on the floor.... The people who delivered after me they were all on the floor. (Hospital delivery)

When I was in the labor room I suffered and stayed until midnight... (After the birth) there was not a bed to put me in. My husband brought a sheet and we just laid down with the baby on the floor. (Hospital delivery)

There were no beds available because of too many people, they brought me two 'domidous' (small mattresses). It's a big hospital I was getting care. God did not let me die. (Hospital delivery)

\section{Lack of respect from the staff}

Women noted they felt mistreated by members of the hospital staff during labour and delivery. They explained how the staff did not tolerate noise or cries during delivery, even making jokes. One woman directly contrasted the privacy of home births with her experience of hospital delivery, noting staff laughed at her pain:

When I give birth at home, no one sees me, and, in the hospital, people make fun of you, they laugh at you. The house is better (laughs!) At home, people do not hear me. The nurses are making fun of me. During my first delivery at HUM, the nurses made fun of me but there were no problems with the delivery. But, after getting pregnant again I thought I was going to give birth at home because I did not really like the experience, it's not for nothing, the only thing I want is to stay at home. (Hospital delivery)

Other women reported being judged by staff because of their physical appearance. They reported that nurses took note of women's clothing and made fun of women who they judged to be 'poor':

Nurses are disrespectful and usually when the poorer are not looking good because they do not have a coat. The poor are humiliated because they do not wear clean clothes. (Hospital delivery)

Some do not have enough financial means, others are afraid because of shame and humiliation of caregivers. (Home delivery)

They should be supervised. The majority of nurses look at poor patients with disgust if they are dirty, poorly dressed. It never 
happened to me, but I have already witnessed this, especially the patients who come from far away. (Hospital delivery)

\section{Hospital-based births are safest}

Women overwhelmingly associated facility-based births with 'safety' and 'security'

Women noted stories of friends and neighbours who had bad experiences and lost their babies during home deliveries. In particular, women acknowledged that delaying going to the hospital after referral by the matron at home could be fatal:

The matron says that the child was 'blocked' in her belly and the matron did not want to keep her and she (the matron) was going back home, and it was too late when a car came to pick her up, she died at the same time as the baby. After my experience at hospital, I advised them to go to hospital. (Hospital delivery)

Women's decisions to give birth at HUM were also driven by family members who encouraged them to pursue hospital births because they were safer. Participants explained that family members, such as husbands and extended family, cautioned them about the dangers of home deliveries. One woman explained how her extended family played a role in planning for their facility-based birth:

Well we have spoken to the family, my mother, my mother in law, sisters and brothers, they all agreed for us to deliver at the hospital, because when you come, if you happened to have any kind of problem, they will notice it and they will take care of it. To stay at your house, wait until the time is due, and never go for checkup and keep on thinking that you are alright, and you have some problems which are going to have some problems with the kid, and all that, they had advised us come to the hospital. (Hospital delivery)

We planned that when I started having pain that he would rush me to the hospital. We planned all of those things. Since the nurses told me not to deliver at home, my partner said he would not let me deliver at home. My partner said if the nurses kept telling me to come deliver at the hospital, maybe they knew something. (Hospital delivery)

Specifically, women noted they felt safer knowing that nurses had specialised knowledge and resources to monitor the progression of labour and make appropriate recommendations to ensure a safe delivery.

\footnotetext{
There is more security at the hospital. As soon as you feel something hurts, there are nurses here that give really good care. But when you are at home, you can die easily. When you come to the hospital, as soon as something hurts, the nurse will take care of it. But when you are home, what can you do? You will just suffer. When you are at the hospital, the nurse consults you, monitors you, cools you down and then she sends you to walk around and once it's time to deliver, you just deliver. But when you are at home, who will cool you down? You have no idea what is going on. Even if someone is helping you push, they can't tell you anything. The person can't tell you anything. (Hospital delivery)
}

Women noted how HUM was able to provide them with medications to manage pain and other medical complications. Women described the use of intravenous solution as a treatment when they became 'weak' during delivery.
Women appreciated the availability of intravenous solution, pills and other injections at the hospital, and they drew sharp contrasts with the lack of these supplies in home births:

Pills can kill you at home; home is a risk; you can get eclampsia and you die. It's a risk if you give birth at home. (Home delivery)

If I had given birth in the hospital, I would be given intravenous solution to replace the blood I had lost. Because I had not given birth at the hospital they did not replace my blood. If I was in the hospital the doctor would give me remedies. Unfortunately, I gave birth at home. (Home delivery)

Women also acknowledged complicated births were best handled at the hospital. In cases of breach presentations, the hospital could perform caesarean section and avoid potentially fatal complications. Women drew a stark contrast between the outcomes of hospital-based deliveries and home-based childbirths for these complicated cases:

I see that it is not a good idea to give birth at home because there can be a bad presentation of the child and the woman may die during childbirth but if it is at the hospital where there are doctors, they could intervene to save the lives of the baby and the mother. (Hospital delivery)

Sometimes the child arrives (by the back) with a breeched presentation and both feet are in front. If you have not died during childbirth, the baby may die during childbirth. If it is in the hospital the doctor will take care of you and give you medicine. (Hospital delivery)

If something is wrong at the hospital they will help you. That's why the hospital is better for you. If you can't deliver the child, they can do it. They can either practice a C-section or give you the scissors. But if you are at home, they won't be able to do anything for you. If you cannot push the child, people at home will not know to give you scissors or the C-section. It is at the hospital that the nurses will know what to do. If you stay at home, you will just die. That's why I would rather come deliver at the hospital. (Home delivery)

\section{DISCUSSION}

We found that pregnant women living in rural Haiti faced substantial barriers related to poverty, such as securing transportation to health facilities far from their homes and costs associated with having a facility-based childbirth. Women also conveyed how traditional birth attendants markedly improved their delivery experience at home; in contrast, the facility environment was perceived to be negative and lacked comparable physical and emotional support. Despite these many barriers, $70 \%$ of women in our study chose to deliver at HUM. Women were eager to prevent complications during birth and have safe deliveries that ensure the well-being of themselves and their babies.

Actualising a facility-based childbirth at HUM required women to overcome numerous obstacles. Many were related to the first two delays: the delay to seek care and the delay in arriving to a health facility. Quantitative analyses revealed that women living further from 
HUM and women with higher likelihood of impoverishment were less likely to deliver at the facility. Qualitative results confirmed that distance, transportation challenges and out-of-pocket costs presented significant barriers for women. Although medical care is provided free-of-charge at HUM, women who delivered at HUM recounted how indirect expenses-including transportation, food for herself and her accompanying caretakers and expenses related to preparing for the arrival of a new baby-could be too costly to afford. Given $>6$ million Haitians live below the poverty line on $<$ US\$2 per day, ${ }^{22}$ it is unsurprising that many barriers for women giving birth in facilities stem from impoverishment. Interventions targeting economic barriers, such as conditional cash transfers to incentivise facility-based childbirths, have increased facility-based childbirths. ${ }^{23}$ However, barriers related to physical and economic accessibility are complex and act in tandem with other factors in the decision to seek care, such as sociocultural factors (eg, education, traditional beliefs) and women's perceived need for care (eg, risk of complications, prior pregnancy) ${ }^{624}$ Thus, economic incentives alone are unlikely to substantially improve uptake of facility-based childbirths. In order to minimise physical and geographic barriers to reaching care, HUM offers ambulance transport; however, demand often exceeds capacity. Additional implementation research may elucidate optimal strategies to reduce the second delay of arriving to a facility.

Despite barriers, pregnant women expressed a desire to give birth at HUM because they were afraid of complications and concerned for safety. Women were educated and aware of the dangers that would require a referral to the hospital for a caesarean section. In fact, qualitative data revealed women who delivered at home believed facility births were safer, reinforcing that knowledge of the benefits of facility-based delivery alone is not sufficient for women to actualise giving birth at HUM.

Health facilities must be prepared to accommodate demand to avoid causing the third delay (ie, the delay in providing adequate care). Women reported inadequate beds and space at HUM, likely driven by the high demand for medical care and scarcity of other comparable facilities in the region. This must be considered in the context of the weakened Haitian health system, within which HUM is inarguably the most equipped and functional in the public sector. Community-level health centres should have the capacity to deliver uncomplicated births. Since the Soins Obstetricaux Gratuits (SOG, Free Obstetrical Care) programme was launched in $2008,{ }^{25}$ obstetric care in Haiti has been free of charge and matrons have been incentivised to refer women with complications to facilities. Accordingly, the number of deliveries in health facilities has increased significantly, ${ }^{25}$ including at HUM where these increases can cause strain on an already fragile system. To ensure safety, health facilities must be prepared with adequate staff, delivery materials and drugs in order to meet the demand of women who are encouraged to come to the facility for delivery.

Qualitative data yielded rich descriptions of the appreciation that women have for 'matrons' (traditional birth attendants) as 'accompagnateurs'. ${ }^{21} 2627$ Women spoke positively about how matrons supported them physically and emotionally through home-based childbirths by providing belly massages and baths, preparing teas and herbal infusions, praying for the baby and performing religious rites. The practices matrons provide during childbirth are considered spiritual and are deeply connected to cultural traditions around the birth of a baby. On the contrary, women described how the absence of support from a matron negatively affected their delivery experience at HUM. Furthermore, women's treatment by staff and delivery practices, such as labouring positions, often left them feeling uncomfortable or ashamed. Women recalled how staff were disrespectful and lacked empathy for their pain and suffering. These same themes have been reported in Haiti and in sub-Saharan Africa. ${ }^{28} 29$ Together, these findings point to the possibility that the presence of the matron in the delivery room alongside midwives may facilitate increased comfort and support for women and discourage the mistreatment of women in pain. Midwives could also serve as a resource on biomedical approaches for matrons, thus mitigating the potential for harms related to traditional birth practices. For example, Walsh et al reported matrons in Haiti recognised that traditional umbilical cord care-practised by matrons and mothers alike-could be unsanitary and that matrons were receptive to using chlorhexidine for the prevention of neonatal sepsis. ${ }^{30}$ Matrons could be an important bridge between the biomedical and sociocultural approaches to care, encouraging women to adopt evidence-based practices before and after birth.

Many of the themes in qualitative data were consistent between women who did and did not return to HUM for delivery. One exception was that qualitative data on hidden costs associated with facility-based deliveries came exclusively from women who had a facility-based delivery. The concordance of themes between groups may be because many women have had multiple deliveries, some of which may have been at home and others at a facility. We referred interview questions to women's most recent pregnancy; however, women's beliefs likely represent the totality of their birthing experiences. Alternatively, women may share their birthing experiences with one another, which can shape perceptions of home-based or facility-based deliveries in the community as a whole.

Our study has several limitations. First, we recruited women from HUM's ANC clinic, thus our cohort comprised entirely of women who received ANC. Given an estimated $91 \%$ of women with a live birth received $\mathrm{ANC}^{31}$ our findings are likely generalisable to the 
majority of pregnant women in Haiti. Factors preventing women from accessing ANC are important for future study and intervention. A second limitation relates to our assessment of facility-based childbirth. If a woman gave birth at a facility other than HUM, we would not have captured this in our study. While we believe delivering at a facility other than HUM was uncommon because women were receiving ANC at HUM, this would result in an underestimate of the per cent of women with a facility-based childbirth and quantitative analyses would reflect factors associated with delivery at HUM rather than factors associated with any facility-based childbirth. Good concordance between qualitative and quantitative findings suggest this theoretical limitation is unlikely to impact study findings or interpretation. Lastly, we cannot rule out the possibility of response bias because the interviewer was affiliated with HUM. Since qualitative interviews yielded some negative feedback, response bias is not a major concern.

In conclusion, pregnant women must choose between receiving physical and emotional support from matrons during home-based childbirths or modern obstetrical care at facilities equipped to handle birth complications. While numerous, complex structural barriers exist, women's negative perceptions related to birthing practices and the environment at health facilities may be a proximal point amenable to intervention. Creating collaborations between matrons and midwives at the facility-level may improve women's uptake of facilitybased childbirth and, ultimately, maternal and neonatal outcomes.

Twitter Molly F Franke @mollyfranke1 and Louise C Ivers @drlouiseivers

Acknowledgements The authors would like to thank the women who participated in this study.

Contributors All authors critically reviewed and approved the final version of the manuscript. Additionally, MR conceived of and designed the qualitative study, collected the qualitative data, conducted qualitative and quantitative analyses and wrote the first draft of the manuscript; CR interpreted the data and provided support in drafting the manuscript; AR contributed to quantitative data analyses and interpreted the quantitative data; GJ contributed to the design of the quantitative study and interpreted the data; AK contributed to all aspects of the qualitative component of the study (design, data collection and interpretation); $\mathrm{HG}$ contributed to all aspects of the qualitative component of the study (design, data collection and analysis); GA oversaw quantitative data collection, conducted quality control of quantitative data and contributed to quantitative data analyses; JPJ oversaw quantitative data collection and quality control of quantitative data, and contributed to quantitative data analyses; MFF conceived of and designed the mixed methods study, oversaw analyses and interpreted the qualitative and quantitative data; $\mathrm{LCl}$ conceived of and designed the quantitative study and interpreted the qualitative and quantitative data.

Funding This study was supported by the United States Centers for Disease Control and Prevention/Unite de Gestion de Projets, Haiti (5U01GGH001060-04) and The Global Health Research Core at Harvard Medical School.

Competing interests None declared.

Patient and public involvement Patients and/or the public were not involved in the design, or conduct, or reporting, or dissemination plans of this research.

Patient consent for publication Not required.

Ethics approval The study protocol for quantitative data collection was approved by Partners Human Research Committee (Boston, Massachusetts, USA; protocol number 2017P000366) and the Haiti National Ethics Committee (Port-au-Prince, Haiti; protocol number 1617-28). The protocol for qualitative data collection was approved by the Harvard Medical School Institutional Review Board (Boston, Massachusetts, USA; protocol number IRB18-0579) and the IRB of Zanmi Lasante (Haiti). All study activities conformed to the principles in the Declaration of Helsinki.

Provenance and peer review Not commissioned; externally peer reviewed.

Data availability statement Data are available upon request. Deidentified participant data are available on request from the corresponding author.

Open access This is an open access article distributed in accordance with the Creative Commons Attribution Non Commercial (CC BY-NC 4.0) license, which permits others to distribute, remix, adapt, build upon this work non-commercially, and license their derivative works on different terms, provided the original work is properly cited, appropriate credit is given, any changes made indicated, and the use is non-commercial. See: http://creativecommons.org/licenses/by-nc/4.0/.

\section{ORCID iD}

Louise C Ivers http://orcid.org/0000-0003-2314-9230

\section{REFERENCES}

1 Trends in maternal mortality: 1990 to 2015: estimates by who, UNICEF, UNFPA, world bank group and the United nations population division. Available: https://data.unicef.org/resources/ trends-maternal-mortality-1990-2015/ [Accessed 12 Jan 2020].

2 Alkema L, Chou D, Hogan D, et al. Global, regional, and national levels and trends in maternal mortality between 1990 and 2015 with scenario-based projections to 2030: a systematic analysis by the un maternal mortality estimation Inter-Agency group. Lancet 2016;387:462-74.

3 Ministry of Public Health and Population. Haïti: 2012 mortality, morbidity, and service utilization survey key findings, 2013. Available: https://dhsprogram.com/pubs/pdf/SR199/SR199.eng.pdf [Accessed 12 Jan 2020].

4 Adegoke AA, van den Broek N. Skilled birth attendance-lessons learnt. BJOG 2009;116 Suppl 1:33-40.

5 Mukherjee JS, Barry DJ, Satti $\mathrm{H}$, et al. Structural violence: a barrier to achieving the millennium development goals for women. $J$ Womens Health 2011;20:593-7.

6 Thaddeus S, Maine D. Too far to walk: maternal mortality in context. Soc Sci Med 1994;38:1091-110.

7 Binder P, Johnsdotter S, Essén B. Conceptualising the prevention of adverse obstetric outcomes among immigrants using the 'three delays' framework in a high-income context. Soc Sci Med 2012;75:2028-36.

8 Combs Thorsen V, Sundby J, Malata A. Piecing together the maternal death puzzle through narratives: the three delays model revisited. PLoS One 2012;7:e52090.

9 Mgawadere F, Unkels R, Kazembe A, et al. Factors associated with maternal mortality in Malawi: application of the three delays model. BMC Pregnancy Childbirth 2017;17:219.

10 Ellis C, Schummers L, Rostoker J-F. Reducing Maternal Mortality in Uganda: Applying the "Three Delays" Framework. Int J Childbirth 2011;1:218-26.

11 Lori JR, Starke AE. A critical analysis of maternal morbidity and mortality in Liberia, West Africa. Midwifery 2012;28:67-72.

12 Waiswa P, Kallander K, Peterson S, et al. Using the three delays model to understand why newborn babies die in eastern Uganda. Trop Med Int Health 2010;15:964-72.

13 Gage AJ, Guirlène Calixte M. Effects of the physical accessibility of maternal health services on their use in rural Haiti. Popul Stud 2006;60:271-88.

14 King-Schultz L, Jones-Webb R. Multi-method approach to evaluate inequities in prenatal care access in Haiti. $J$ Health Care Poor Underserved 2008;19:248-57.

15 Barnes-Josiah D, Myntti C, Augustin A. The "three delays" as a framework for examining maternal mortality in Haiti. Soc Sci Med 1998;46:981-93.

16 Creswell JW. Steps in conducting a scholarly mixed methods study, 2013: 48.

17 Schreiner M. Simple poverty Scorecard® Poverty-Assessment tool Haiti, 2016.

18 itchler M, Ballard T, Swindale A, et al. Introducing a simple measure of household hunger for cross-cultural use. Washington, DC: Food and Nutrition Technical Assistance II project, AED, 2011. http://www. fao.org/fileadmin/user_upload/wa_workshop/docs/HH_Hunger_ Scale.pdf

19 Miettinen OS. Theoretical epidemiology. principles of occurrence research in medicine. New York: Wiley, 1985. 
20 Hsieh H-F, Shannon SE. Three approaches to qualitative content analysis. Qual Health Res 2005;15:1277-88.

21 Farmer P. Partners in help: assisting the poor over the long term, 2011.

22 The World Bank. Haiti-Overview. Available: https://www.worldbank. org/en/country/haiti/overview [Accessed 12 Jan 2020].

23 Glassman A, Duran D, Fleisher L, et al. Impact of conditional cash transfers on maternal and newborn health. J Health Popul Nutr 2013;31:S48-66.

24 Gabrysch S, Campbell OMR. Still too far to walk: literature review of the determinants of delivery service use. BMC Pregnancy Childbirth 2009;9:34.

25 World Health Ogranization. Free obstetric care in Haiti making pregnancy safer for mothers and newborns, 2010. Available: https:// apps.who.int/iris/bitstream/handle/10665/70441/WHO_MPS_10. 05_eng.pdf;jsessionid=3FFB9328473046C7489C3FE9C61C1A99? sequence $=1$ [Accessed 12 Jan 2020].
26 Palazuelos D, Farmer PE, Mukherjee J. Community health and equity of outcomes: the partners in health experience. Lancet Glob Health 2018;6:e491-3.

27 Behforouz HL, Farmer PE, Mukherjee JS. From directly observed therapy to accompagnateurs: enhancing AIDS treatment outcomes in Haiti and in Boston. Clin Infect Dis 2004;38 Suppl 5:S429-36.

28 Dev A, Kivland C, Faustin M, et al. Perceptions of isolation during facility births in Haiti - a qualitative study. Reprod Health 2019;16:185.

29 Moyer CA, Mustafa A. Drivers and deterrents of facility delivery in sub-Saharan Africa: a systematic review. Reprod Health 2013;10:40.

30 Walsh S, Norr K, Sankar G, et al. Newborn cord care practices in Haiti. Glob Public Health 2015;10:1107-17.

31 Institut Haïtien de l'Enfance - IHE and ICF. Enquête Mortalité, Morbidité et Utilisation des Services - EMMUS-VI 2016-2017 PétionVille, Haïti, et Rockville, Maryland, USA : IHE et ICF, 2018. https:// www.dhsprogram.com/pubs/pdf/FR326/FR326.pdf 